\title{
Chronic Food Insecurity and Malnutrition in Moroto: Karamoja Sub Region Still Highly Attributed to Poor Water and Sanitation Practices
}

\section{Andrew Omuna*}

Communications and Advocacy, IBFAN Uganda, Kampala, Uganda

The problem of undernutrition resulting from both macro- and micro-nutrient (vitamin and mineral) deficiencies as well as overnutrition is increasingly existing side by side across the world. About 795 million people are under-nourished or food deprived globally and specific to Uganda, the 2011 Uganda Demographic and Health Survey shows that $5 \%$ of children under 5 years are wasted, $14 \%$ underweight and 33\% stunted. The consequences of undernutrition are diverse, ranging from poor physical growth and mental development, devastating illness and death, culminating into incalculable loss of human potential, social and economic development.

In June 2017, World Food Program (WFP) contracted the International Baby Food Action Network (IBFAN) Uganda to conduct the Food Security and Nutrition Assessment in the Karamoja subregion covering all the 7 districts of Abim, Amudat, Kaabong, Kotido, Moroto, Nakapiripirit and Napak. The assessment revealed that these districts were highly affected by chronic food insecurity coupled with high levels of malnutrition that were of public health concern. Overall, Food Security classification showed nearly half (46\%) of the households in Karamoja sub-region were food insecure while the prevalence of wasting was $13.8 \%$ which according to WHO threshold is 'serious/high'.

The reported levels of malnutrition were attributed to different factors related to gender of household head, mother's education level as well as nutritional status, Illness in the child, quantity of water used per person per day, access to toilets, food consumption patterns, livestock ownership, coping strategies and food security situation. Poor water and sanitation practices were among the critical factors that stood out affecting the entire sub region especially Moroto district. Water and sanitation practices assessed included access to water sources, quantity of water used, treating of drinking water, availability of toilet facilities, and type of toilet facilities.

Almost all households in Moroto district had access to relatively safe water that included boreholes, piped water through taps and protected wells/springs with only Katekile subcounty where one third of households accessed water from unsafe sources and therefore had higher prevalence of malnutrition of $22.2 \%$ compared to the other subcounties in the district. The high coverage of safe water was because of partners like UNICEF that sunk more boreholes, though "as a district we are aware, we are not yet where we want to be because most boreholes had broken down and the functional ones had little or no water due to low water tables".

Treating of drinking water was not a common practice in the district by $21 \%$ of households. The main method of water treatment was by boiling at $78 \%$, which should not underscore the importance of ensuring access to relatively safe sources of household water. The recommended amount of water for basic household hygiene and sanitation is at least 15 litres per person per day (pppd). However, only $19 \%$ of households in the district were using the recommended amount of water pppd.

Latrine coverage (58\%) and utilization was observed to still be a challenge in the district despite partner involvement in providing support to establish latrines and sensitize communities on their use. Nevertheless, this could be attributed to lower than expected geographical coverage by the partners. Among those with toilets, the open pit without a superstructure was the main type of facility for $65 \%$ of households. Poor hygiene and sanitation practices could be attributed to strong cultural beliefs related to latrine use.

About eight in ten households in the district reported that their children were sick in the 2 weeks prior to the survey attributing this to the poor water and sanitation situation. Diseases and/or illnesses cause loss of appetite, feeling sick, vomiting and/or changes in bowel habit (such as diarrhea) which in turn result into low or no absorption of nutrients by the body to promote good health.

Despite the high numbers of support organizations engaging the district with diverse activities focused on raising the profile of water and sanitation and other key related issues, the current levels of malnutrition still demand for simultaneous or integrated nutrition and sanitation sensitization programs geared towards support to households in adopting proper water and sanitation practices, and other related issues.

In such situations, securing and maintaining a high level of nutrition commitment that translates into tangible action, resources and results is widely recognized as essential for scaling up the support for nutrition with key emphasis and highlight directed to challenges; relating to water and sanitation. Mobilization of partners to work with district structures is critical for building this commitment and ensuring that evidence and experiences of best WATSAN practices are shared. In addition, inspiring the people of Karamoja to play their part by working together to address the challenges facing them is crucial for success to be gained.

Streamlining professional capacity for nutrition coupled with community sensitization on the usage and importance of proper water and sanitation programs in Moroto district like in many countries with high levels of malnutrition should inspire international development programs to create solutions to overcome the gap in the short-term. This may call for designing and implementing comprehensive training packages, such as the WASH and integrating it into the Infant and Young Child Feeding Counseling support with clear guidance on how to adapt them and enable the local government and NGOs to provide in-service training in the district together with the existing national experts.

${ }^{*}$ Corresponding author: Andrew Omuna, Communications and Advocacy, IBFAN Uganda, Kampala, Uganda, Tel: +256705134433; E-mail: andrewc4d.org@gmail.com

Received September 27, 2017; Accepted October 20, 2017; Published October 23, 2017

Citation: Omuna A (2017) Chronic Food Insecurity and Malnutrition in Moroto: Karamoja Sub Region Still Highly Attributed to Poor Water and Sanitation Practices. J Mass Communicat Journalism 7: 352. doi: 10.4172/2165-7912.1000352

Copyright: (c) 2017 Omuna A. This is an open-access article distributed under the terms of the Creative Commons Attribution License, which permits unrestricted use, distribution, and reproduction in any medium, provided the original author and source are credited. 